\title{
Kinesthetic and Visual Control of a Bimanual Task: Specification of Direction and Amplitude
}

\author{
Martha Flanders ${ }^{\mathrm{a}}$ and Paul J. Cordo \\ Neurological Sciences Inștitute, Good Samaritan Hospital and Medical Center, Portland, Oregon 97209
}

\begin{abstract}
Kinesthetic information āboùt à perturbation can quickly modify motor activity by producing reflexive compensation. The purpose of the present study was to determine how quickly kinesthetic information about target movement can modify motor activity. Visual information about target movement is known to guide motor activity both quickly and accurately. Therefore, we compared the speed and accuracy of responses to kinesthetically and visually presented targets.
\end{abstract}

Human subjects produced changes in elbow torque as quickly and accurately as possible after the random presentation of 1 of 8 target torques. Information about the direction and amplitude of the target torque was provided either kinesthetically or visually. Responses to kinesthetic targets started at an average latency of $150 \mathrm{msec}$, and after an additional $159 \mathrm{msec}$, these responses became accurately graded according to target amplitude. Responses to visual targets started at an average latency of $250 \mathrm{msec}$, and after an additional $208 \mathrm{msec}$, these responses became accurately graded according to target amplitude. The accuracy of responses to kinesthetic targets was very similar to the accuracy of responses to visual targets.

We conclude that the neural processing of kinesthetic information about target movement is sufficiently fast and accurate to guide typical motor activities.

The human CNS receives kinesthetic input from muscle spindles, tendon organs, and joint and cutaneous receptors. One function of kinesthetic input is to compensate for perturbations to ongoing motor activity. For example, if a limb is perturbed during movement to a stationary target, stretch and unloading reflexes can quickly modify motor activity so as to resist the perturbation. Another function of kinesthetic input is to initiate and parameterize new motor commands. For example, if target movement is sensed kinesthetically, kinesthetic input can be used to select the direction and amplitude of a new motor command that is appropriate for attaining the new target.

It is well established that kinesthetic information about a perturbation can produce reflexive compensation within 20-100 msec. Because this reflex mechanism works very quickly, it is

\footnotetext{
Received Jan. 25, 1988; revised June 3, 1988; accepted July 18, 1988

This work was supported hy National Research Service Award NS07661, and United States Public Health Service Grant AR31017. We thank Dr. Susan P. Moore for consultation.

Correspondence should be addressed to Dr. Paul J. Cordo, Neurological Sciences Institute, Good Samaritan Hospital and Medical Center, 1120 N.W. 20th Avenue, Portland, OR 97209.

${ }^{a}$ Present address: Department of Physiology, University of Minnesota, Minneapolis, MN 55455.

Copyright (C) 1989 Society for Neuroscience $0270-6474 / 89 / 020447-07 \$ 02.00 / 0$
}

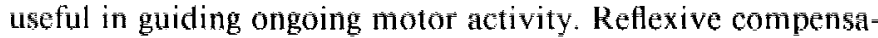
tion can occur cither at the joint that is perturbed (autogenic compensation) or at a diferent joint (nonautogenic compen= sation). Autogenic compensation was first reported by Ham= mond (1954) and has been described by many other investigators since then (e.g, Crago et âl., 1976; Marsden et al., 1976; Dufresne et al., 1978). There arc scveral examples of nonautogenic compensation in more recent literature: during speech, perturbation of the lower lip results in a compensatory response of the upper lip (Abbs and Gracco, 1984); during pinching movements, perturbation of the thumb results in a compensatory response of the forefinger (Cole and Abbs, 1987); and when a grasped object begins to slip, cutaneous input automatically produces a more forceful grip (Johansson and Westling, 1984, 1987).

It is also well established that kinesthetic information about target movement can be used to parameterize new motor commands, but the speed of this mechanism is not known. Therefore, it is unclear how useful this mechanism is in guiding typical motor activities. It has been demonstrated that, if a motor response has already been parameterized in advance of a kinesthetic triggering stimulus, the response can be initiated about $110 \mathrm{msec}$ after the stimulus is presented (Chernikoff and Taylor, 1952; Evarts and Vaughn, 1978; Flanders, et al., 1986). It has also been demonstrated that subjects can accurately use kinesthetic information to parameterize new motor commands: subjects can use kinesthetic input to match the position or velocity of one arm, with the contralateral arm (e.g., Sittig et al., 1985, 1987; De Domenico and McCloskey, 1987). However, since there was no time constraint placed on the production of these matching responses, it remains unclear how much time is required for kinesthetic input to parameterize new motor commands.

In the present study, we tested the hypothesis that kinesthetic information about target location can quickly and accurately parameterize new motor commands. In order for kinesthetic input to be useful in guiding a limb toward a moving target, new motor commands must be parameterized both quickly and accurately. Because visual information about target movement is known to be used both quickly and accurately (Pélisson et al., 1986; Cordo, 1987; Gordon and Ghez, 1987), we compared responses to visually and kinesthetically presented targets. We compared the speed with which subjects began to respond in the correct direction, the speed with which response amplitude became related to target amplitude, and the overall accuracy of the responses.

\section{Materials and Methods}

Six normal human subjects (ages 23-49) participated in this experiment. In the first half of the experiment, each subject responded to a visually 

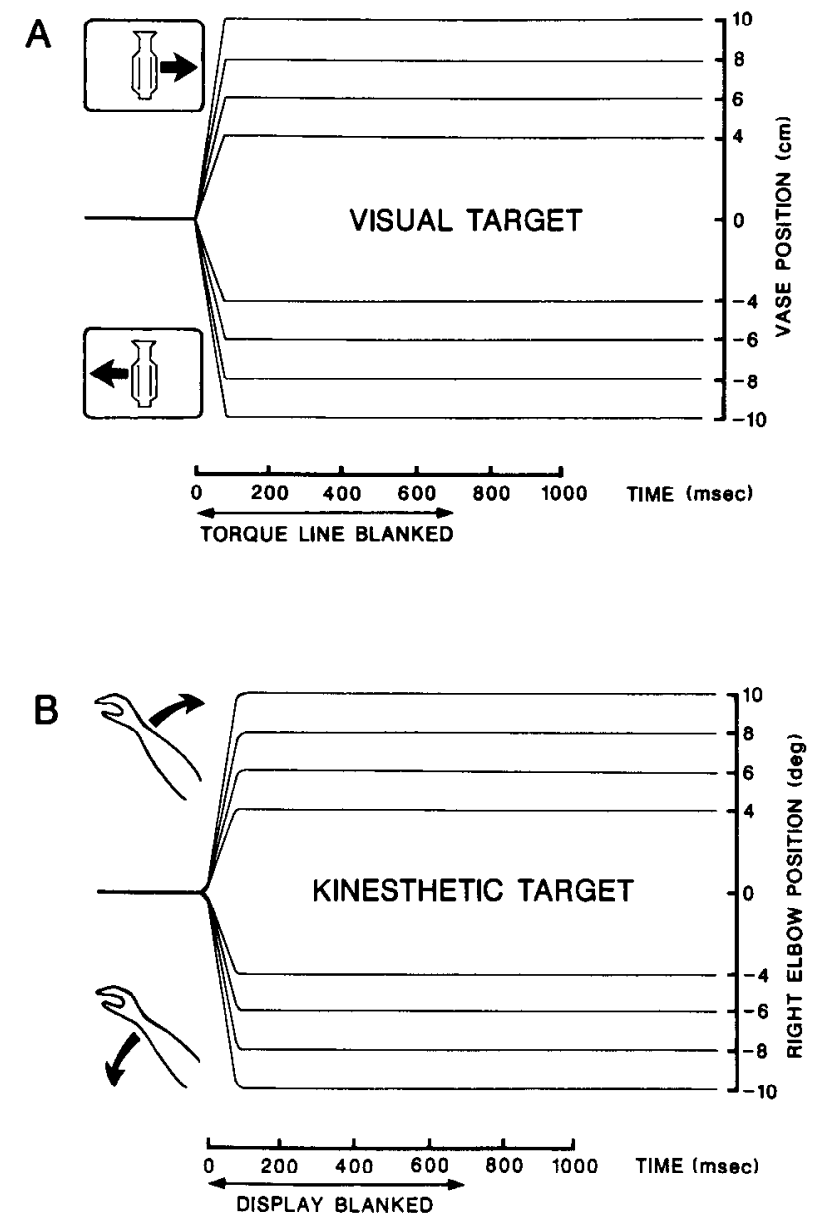

Figure 1. Target presentation. Target position across time was traced from recorded data. $A$, In the first half of the experiment, the lines representing left and right elbow torque were blanked (at $0 \mathrm{msec}$ ) and the vase was moved to 1 of 8 positions. The line representing left elbow torque reappeared at $700 \mathrm{msec} . B$. In the second half of the experiment, the visual display was blanked (at $0 \mathrm{msec}$ ) and the right elbow was rotated to 1 of 8 positions. The vase and the line representing left elbow torque reappeared at $700 \mathrm{msec}$

presented target with a fast and accurate change in torque at the left elbow. After a rest period of about a half-hour, the subject returned to the apparatus and responded to a kinesthetically presented target with the same type of motor response. The speed and accuracy of responses to visual and kinesthetic targets were then compared.

Apparatus. Each subject sat at a table and faced a $28 \times 40 \mathrm{~cm}$, vector graphics, visual display. The visual display was about 1 meter in front of the subject, at eye level. The subject placed the left and right forearms on a horizontal surface, with each wrist in a stationary wrist cuff. In order to keep the subject's elbows isometric, we provided postural support at each elbow, and in front of the chest. The angular position of each elbow (measured in the horizontal plane) was $60^{\circ}$ extended from a line parallel to the chest.

By producing flexion torque at each isometric elbow, the subject controlled the positions of 2 of the graphics objects that were projected on the visual display. Elbow torque was transduced by strain gauges, which were cemented to the base of each wrist cuff. The torque signals from the left and right elbows were used to control the horizontal positions of 2 vertical lines. When the subject produced no torque, these 2 lines were at the extreme left and right margins of the display. When the subject produced flexion torque at the left elbow, the left line moved to the right, and when the subject produced flexion torque at the right elbow the right line moved to the left. A $0.75 \mathrm{Nm}$ change in torque moved the line $1 \mathrm{~cm}$.

The experimenter controlled the position of 2 target zones, which were also projected on the visual display. These target zones were at the right and left borders of a $4 \mathrm{~cm}$ wide vase (see Fig. $1 A$ ). Each target zone was $1 \mathrm{~cm}$ wide and therefore represented a $0.75 \mathrm{Nm}$ range of elbow torque.

The experimenter also controlled the angular position of the right elbow. Rotation of the right elbow was accomplished by a servocontrolled hydraulic connection to the right wrist cuff. The angular position of the right elbow was transduced by a precision potentiometer. The right arm was screened from the subject's view.

Experimental protocol. Before each part of the experiment, the subject was given 1-3 practice trials with each target position. After this practice period, we observed no further improvement in performance. The kinesthetic task required more practice prior to steady-state performance. This was because with the kinesthetic task, the sensory modalities of the target and the knowledge of results were different. It was necessary for subjects to learn the correspondence between right elbow angle and vase position. The kinesthetic part of the experiment was placed after the visual part of the experiment to facilitate this practice.

For all trials in both parts of the experiment, the subject began the trial with a preparatory task, termed "holding the vase": The subject held the left and right lines in the left and right target zones by producing $8 \mathrm{Nm}$ of flexion torque with each isometric elbow.

In the first half of the experiment, the target level of torque was visually presented. After 2-3 sec of "holding the vase," the right and left lines disappeared and the vase moved (Fig. 1 $A$ ). The vase (i.e., the target) moved either to the right or to the left and stopped at 1 of 8 target positions. The total duration of the target movement was $70 \mathrm{msec}$. Each target position was presented 10 times in an experimental session. All 80 trials were randomized to prevent the subject from anticipating the direction and amplitude of target movement.

In the second half of the experiment, the target level of torque was kinesthetically presented. After 2-3 sec of "holding the vase," the entire visual display was blanked and the right elbow was rotated (Fig. 1B). The elbow was either extended away from the subject or flexed toward the subject and stopped at 1 of 8 target positions. As in the first half of the experiment, the total duration of target movement was $70 \mathrm{msec}$. Each target position was presented 10 times, and all 80 trials were randomized.

The subject was instructed to respond to the visually or kinesthetically presented target by changing the amount of flexion torque at the left elbow. When the vase was moved to the right by $4,6,8$, or $10 \mathrm{~cm}$ and when the right elbow was extended by $4^{\circ}, 6^{\circ}, 8^{\circ}$, or $10^{\circ}$, the subject was required to increase left elbow torque to $11.0,12.5,14.0$, or $15.5 \mathrm{Nm}$, respectively (see Fig. 3 ). When the vase was moved to the left by 4,6 , 8 , or $10 \mathrm{~cm}$, or when the right elbow was flexed by $4^{\circ}, 6^{\circ}, 8^{\circ}$, or $10^{\circ}$, the subject was required to decrease left elbow torque to $5.0,3.5,2.0$, or $0.5 \mathrm{Nm}$, respectively (see Fig. 3).

Each subject was instructed to respond to the target movement as quickly and as accuratcly as possible. Beginning $700 \mathrm{msec}$ after the onset of the vase movement or elbow rotation, the subject was informed about the success of his or her response. With the visual target, this knowledge of results was given by the reappearance of the line representing left elbow torque. With the kinesthetic target, both the vase and the left line reappeared. When the vase reappeared, its position corresponded to the position of the right arm (for example, the vase was $8 \mathrm{~cm}$ to the right when the arm was extended by $8^{\circ}$ ). For both the visual and the kinesthetic tasks, the subject was encouraged to have the left line in the left target zone when the line reappeared.

When the left line reappeared, the subject was required to correct any errors by moving the line into the target zone. The subject was always required to hold the left line in the target zone until the end of the trial was signaled by the blanking of the display, about $2 \mathrm{sec}$ after response onset. The subject was not instructed to make any particular response with the right elbow, and the right line did not reappear for an additional $5-10 \mathrm{sec}$.

Data collection and analysis. A Digital Equipment Corporation (PDP. 11) computer was used to control the movement of the vase on the visual display and the angular position of the right elbow, as well as to record and analyze electromyographic (EMG) and mechanical data. All data were sampled at $500 \mathrm{~Hz}$.

We used EMG data to determine the pattern of agonist-antagonist activation our subjects used to produce their torque responses. We collected and analyzed EMG data from the left biceps (agonist) and triceps (antagonist) muscles. Muscle activity was recorded using bipolar $\mathrm{Ag} / \mathrm{AgCl}$ surface electrodes $(1.3 \mathrm{~cm}$ diameter, $5 \mathrm{~cm}$ apart). $\mathrm{EMG}$ signals were preamplified $(100 \times)$ at the recording site, and then further am- 

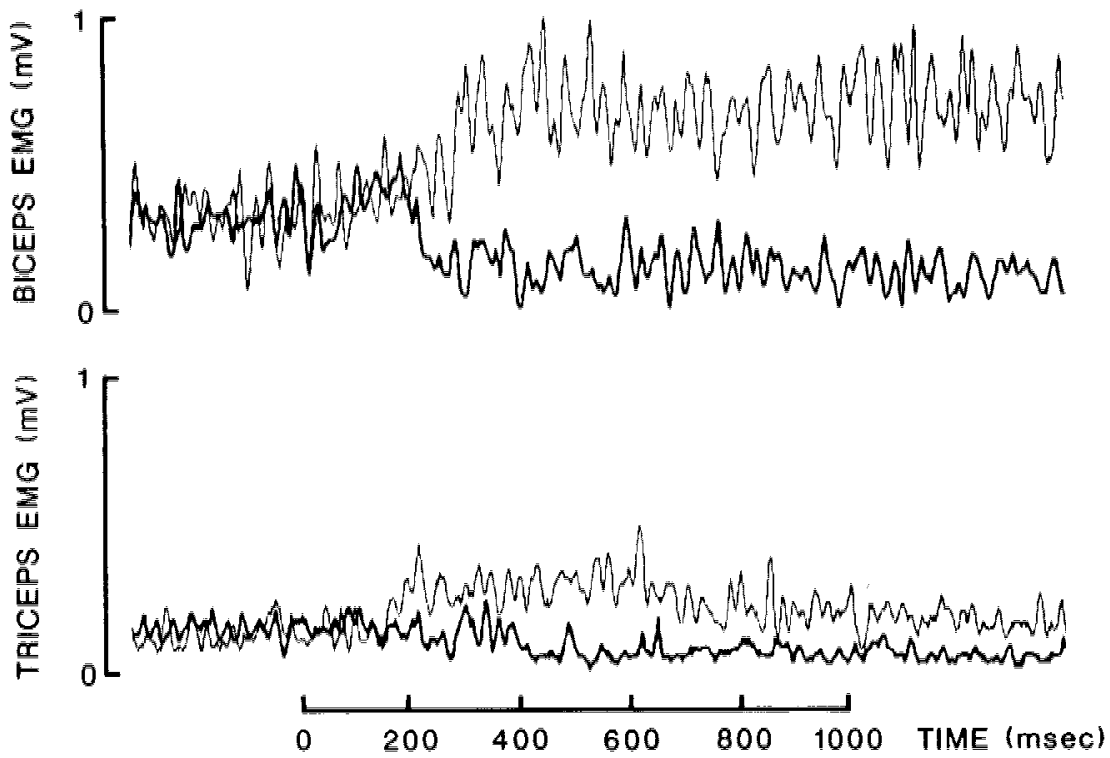

Figure 2 . Coactivation of antagonistic muscles. During the pieparatory task, the subject produced $8 \mathrm{Nm}$ of flexion torque by activating biceps and coactivating triceps to a lesser extent. At 0 msec, a visually presented target signaled the subject to produce a different level of torque. The thin traces are the muscle activity that produced

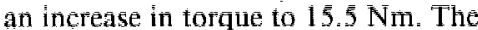
thick traces are the muscle activity that produced a decresca in torgue to 0.5 $\mathrm{Nm}$. Each trace is the average of 10 trials from a single subject (M.F.). Data from each trial were aligned at the onset of the target presentation ( $0 \mathrm{msec}$ ). plified, bandpass-filtered $(80-5000 \mathrm{~Hz})$, full-wave rectified, and smoothed with a time constant of $10 \mathrm{msec}$.

We used torque data from the left elbow to calculate the speed and accuracy of responses to visual and kinesthetic targets. Sampling of torque data began $300 \mathrm{msec}$ prior to the onset of the vase or elbow movement and continued for $2.5 \mathrm{sec}$. The onset of each torque response was visually determined and measured with a graphics cursor program. We used other computer programs to average torque records and to analyze response accuracy.

In about $3 \%$ of all trials, subjects began to respond in the wrong direction but quickly corrected their responses. Trials with directional errors were not included in the data analysis.

\section{Results}

Muscle activity

For both the visual and the kinesthetic tasks, the left biceps and triceps were coactivated (Flanders and Cordo, 1987). Biceps and triceps EMG records from a representative subject are shown in Figure 2 for responses to visual targets. The thin traces are averages from the 10 trials in which a $7.5 \mathrm{Nm}$ increase in torque was required; the thick traces are averages from the 10 trials in which a $7.5 \mathrm{Nm}$ decrease in torque was required. Increases in flexion torque were produced by increases in both biceps activity and triceps activity. Decreases in flexion torque were produced by decreases in both biceps activity and triceps activity. Responses to kinesthetic targets were produced by the same pattern of coactivation.

\section{Torque responses}

We compare torque responses to visual and kinesthetic targets to demonstrate similarities in the control of response direction and amplitude and similarities in response accuracy. The averaged torque responses of all 6 subjects are shown in Figure 3, where each trace is the grand mean of responses to a given target amplitude. Responses to visual targets are shown in Figure $3 \mathrm{~A}$, whereas responses to kinesthetic targets are shown in Figure $3 B$. In both Figures $3 A$ and $3 B$, the initial level of left elbow torque $(8 \mathrm{Nm})$ and the 8 target levels of torque $(0.5-15.5 \mathrm{Nm})$ are shown on the ordinate. Figure 3 illustrates the 3 features of these torque responses that were of interest in this study: (1) the latency of response onsets, (2) the time at which the response amplitude became significantly related to the target amplitude, and (3) response accuracy.

\section{Response latency}

For visual targets, subjects began to respond at an average of $250 \mathrm{msec}$ after the onset of vase movement. The beginning and

Table 1. Kinesthetic and visual control

\begin{tabular}{|c|c|c|c|c|c|}
\hline \multirow[b]{2}{*}{ Subject } & \multicolumn{2}{|c|}{ Torque increases } & \multicolumn{2}{|c|}{ Torque decreases } & \multirow[b]{2}{*}{$\begin{array}{l}\text { Success } \\
\text { rate } \\
(\%) \\
\end{array}$} \\
\hline & $\begin{array}{l}\text { Response } \\
\text { latency } \\
\text { (msec) }\end{array}$ & $\begin{array}{l}\text { Sig. } \\
\text { slope } \\
\text { (msec) } \\
\end{array}$ & $\begin{array}{l}\text { Response } \\
\text { latency } \\
(\mathrm{msec})\end{array}$ & $\begin{array}{l}\text { Sig. } \\
\text { slope } \\
(\mathrm{msec}) \\
\end{array}$ & \\
\hline \multicolumn{6}{|c|}{ Visual targets } \\
\hline J.B. & $274(8)$ & 90 & $262(5)$ & 160 & 15 \\
\hline M.F. & $191(5)$ & 200 & $233(6)$ & 320 & 20 \\
\hline A.F. & $186(6)$ & 40 & $200(8)$ & 170 & 24 \\
\hline R.K. & $272(13)$ & 400 & $245(8)$ & 130 & 13 \\
\hline B.R. & $285(10)$ & 100 & $295(8)$ & 180 & 31 \\
\hline S.S. & $\underline{245}(7)$ & $\underline{210}$ & $315(7)$ & $\underline{500}$ & 13 \\
\hline $\bar{X}$ & 242 & 173 & 258 & 243 & 19 \\
\hline $\mathrm{SE}$ & 18 & 53 & 17 & 58 & 3 \\
\hline \multicolumn{6}{|c|}{ Kinesthetic targets } \\
\hline J.B. & $169(8)$ & 50 & $154(8)$ & 160 & 31 \\
\hline M.F. & $118(6)$ & 100 & $151(9)$ & 300 & 24 \\
\hline A.F. & $108(3)$ & 70 & $132(7)$ & 50 & 11 \\
\hline R.K. & $175(14)$ & 130 & $131(5)$ & 70 & 18 \\
\hline B.R. & $149(6)$ & 30 & $202(15)$ & 440 & 25 \\
\hline S.S. & $\underline{172}(11)$ & 250 & $136(8)$ & 260 & $\underline{21}$ \\
\hline $\bar{X}$ & $149^{a}$ & $105^{b}$ & $151^{c}$ & $213^{b}$ & $22^{b}$ \\
\hline SE & 12 & 32 & 11 & 61 & 3 \\
\hline
\end{tabular}

Averages from individual subjects and grand means $(\bar{X})$ and standard errors (SE) from all subjects are tabulated for torque increases (left) and torque decreases (right) made in response to visual and kinesthetic targets. The average response latency of each subject is shown along with the SE for 40 trials. The latency of significant amplitude control ("Sig. slope") is the time from response onset to a significant regression slope.

${ }^{a}$ Significantly different from visual targets at $p<0.01$.

${ }^{b}$ Not significantly different from visual targets.

c Significantly different from visual targets at $p<0.001$. 

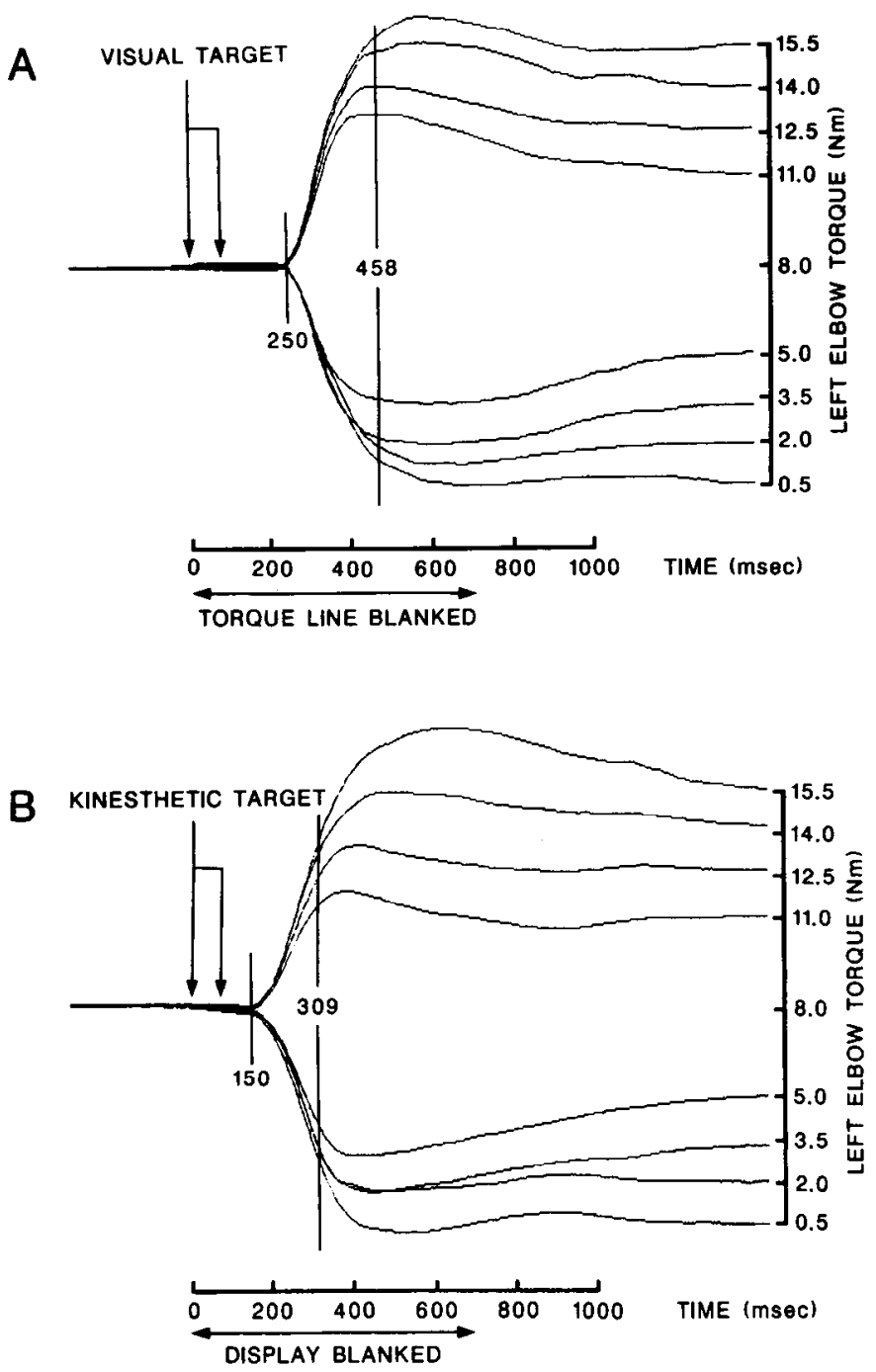

Figure 3. Torque responses. $A$, In the first half of the experiment, the vase (visual target) started to move at $0 \mathrm{msec}$ and reached its final position after $70 \mathrm{msec}$, as shown by the 2 vertical lines with arrows. On average, subjects began to respond at $250 \mathrm{msec}$. Response amplitude became significantly related to the target amplitude at $458 \mathrm{msec} . B$, In the second half of the experiment, the right elbow (kinesthetic target) started to rotate at $0 \mathrm{msec}$ and reached its final position after $70 \mathrm{mscc}$. Average response latency was $150 \mathrm{msec}$. Response amplitude became significantly related to the target amplitude at $309 \mathrm{msec}$. Each trace is the grand mean response of 6 subjects.

end of vase movement are shown by 2 vertical arrows in Figure $3 \mathrm{~A}$. Individual torque responses were aligned at response onset for averaging, and the onset of the averages was placed $250 \mathrm{msec}$ after the onset of vase movement.

The average latencies of responses to visual targets are shown in the top half of Table 1, which includes the average response latencies for each subject, as well as the grand mean response latencies for all subjects. Response latencies were calculated separately for responses involving increases and decreases in torque. On average, increases in torque started $242 \mathrm{msec}$ after the onset of the vase movement, whereas decreases in torque started $258 \mathrm{msec}$ after the onset of the vase movement. Latencies for increases and decreases in torque were not significantly different from each other $[t(10)=0.65, p>0.05]$.

For kinesthetic targets, subjects began to respond in the cor- rect direction at an average of $150 \mathrm{msec}$ after the onset of the right elbow rotation. In Figure $3 B$, the onsets of all responses are aligned at $150 \mathrm{msec}$. The average latencies of responses to kinesthetic targets are shown in the bottom half of Table 1. Increases in torque started $149 \mathrm{msec}$ after the onset of the elbow rotation, whereas decreases in torque started $151 \mathrm{msec}$ after the onset of the elbow rotation. These response latencies were not significantly different from each other $[t(10)=0.12, p>0.05]$.

Responses to kinesthetic targets began significantly sooner than responses to visual targets. For increase in torque, this difference in latency was significant at the $p<0.01$ level $[t(10)$ $=4.37$, whereas for decreases in torque, this difference in latency was significant at the $p<0.001$ level $[t(10)=5.29]$.

\section{Control of response amplitude}

We used a linear-regression technique to determine the time at which the response amplitude became significantly related to the target amplitude. The actual level of left elbow torque was plotted against the target level of left elbow torque, using the individual trials of each subject. A new plot was made at every $10 \mathrm{msec}$ interval, beginning at response onset. Separate plots were made for responses involving increases and decreases in torque. Plots at several different times after response onset are shown in Figure 4 for the increases in torque produced by a representative subject.

Shortly after the onset of responses to visual targets, the slope of the regression line was near zero. In Figure $4 A$, the plot on the left contains data from $10 \mathrm{msec}$ after the onset of responses to visual targets (solid circles). At this time, the subject had increased torque to slightly more than the $8.0 \mathrm{Nm}$ preparatory level (open square), but this torque was not related to the target level (slope $=0.005$ ). At $10 \mathrm{msec}$ after response onset, the variahility of response amplitude was small.

At $90 \mathrm{msec}$ after the onset of responses to visual targets, the slope of the regression line became significantly greater than zero, as shown by the middle plot in Figure $4 A$ (slope $=0.152$ ). We determined the statistical significance of the slope using a 1 -tailed $t$-test (Snedecor and Cochran, 1971), with $p<0.05$. This statistical technique takes into account both the slope of the regression line and the variability of response amplitude. This technique provides a conservative estimate of the earliest time that target amplitude information may have been incorporated into responses.

The earliest time at which the slope was significantly greater than zero was determined for each subject. For responses to visual targets, these times are listed in the top half of Table 1. The grand mean time from response onset to the earliest time at which the slope was significant was $173 \mathrm{msec}$ for increases in torque and $243 \mathrm{msec}$ for decreases in torque. These grand means were not significantly different from each other $[t(10=$ $0.89, p>0.05]$.

Plots of data from responses to kinesthetic targets were very similar to those from responses to visual targets. Plots from 3 different times during responses to kinesthetically presented targets are shown in Figure $4 B$. At $10 \mathrm{msec}$ after response onset, the slope of the regression line was close to zero (Fig. $4 B$, left plot, slope $=-0.004$ ). The slope of the regression line became significantly greater than zero at $50 \mathrm{msec}$ after response onset (Fig. $4 B$, middle plot, slope $=0.098$ ). As shown in the bottom half of Table 1, the grand mean time from response onset to the earliest time at which the slope was significant was $105 \mathrm{msec}$ for increases in torque and $213 \mathrm{msec}$ for decreases in torque. 

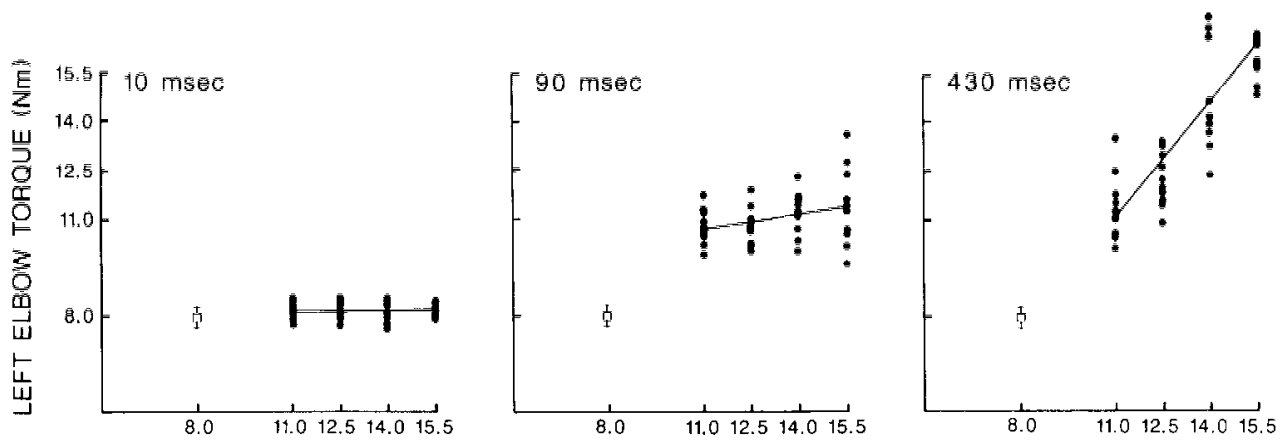

TARGET LEVEL OF LEFT ELBOW TORQUE (Nm)

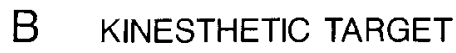

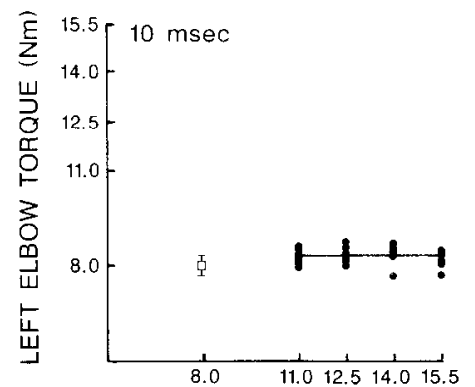
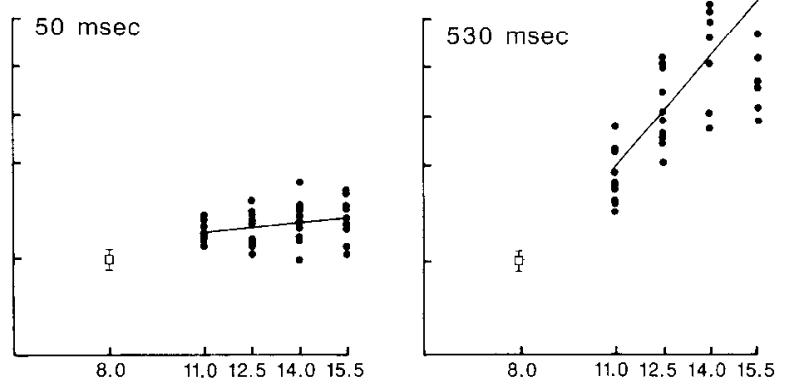

TARGET LEVEL OF LEFT ELBOW TORQUE (Nm)
Figure 4. Linear-regression analysis of torque responses. The actual level of left elbow torque is plotted against the target level of left elbow torque at various times after response onset. Each solid circle represents an individual trial. $A$, Responses to visual targets. At 10 , 90 , and $430 \mathrm{msec}$ after response onset, the slopes were $0.005,0.152$, and 1.198 , respectively. $B$, Responses to kinesthetic targets. At 10, 50, and $530 \mathrm{msec}$ after response onset, the slopes were $-0.004,0.098$, and 1.162 , respectively. All data are from the same subject (I.B.). The open squares represent the average of 40 trials (with SE bars) at 10 msec before response onset.
These grand means were not significantly different from each other $[t(10)=0.89, p>0.05]$.

The earliest time at which the slope of the relationship between response amplitude and target amplitude is significantly greater than zero is an estimate of the onset of significant amplitude control. The time from the onset of the torque response to the onset of significant amplitude control was not significantly different for responses to visual and kinesthetic inputs $[t(10)=$ 1.10 for increases in torque, $t(10)=0.36$ for decreases in torque, $p>0.05$, Table 1].

For pooled data from both increases and decreases in torque, the average time from the onset of the target presentation to the onset of significant amplitude control was $458 \mathrm{msec}$ for responses to visual targets (Fig. $3 A$ ) and $309 \mathrm{msec}$ for responses to kinesthetic targets (Fig. $3 B$ ). These 2 latency estimates were significantly different from each other $[t(10)=2.48, p<0.05]$.

\section{Response accuracy}

The average accuracy of responses to visual and kinesthetic targets was very similar (Fig. 3). The regression analysis described above produced an estimate of the onset of significant amplitude control. Although subjects began to control amplitude early in their responses, the task did not require accuracy until $700 \mathrm{msec}$ after the onset of target presentation, the moment that knowledge of results was given. We analyzed the accuracy of responses at $700 \mathrm{msec}$ after the onset of target presentation.

The average accuracy and the variability of individual responses to visual targets are illustrated in the right plot of Figure $4 A$. This plot contains data from $700 \mathrm{msec}$ after the onset of vase movement (on average, $430 \mathrm{msec}$ after response onset). For this subject, the slope of the relationship between response amplitude and target amplitude was close to unity (slope = 1.198). A slope of unity would indicate that during an average response, the left line reappeared in the target zone. Because the target zone was very small $(0.75 \mathrm{Nm})$, the line reappeared in the target zone in only a small percentage of all trials. The percentage of successful responses to visual targets is listed for each subject in the top of Table 1 , in the right-most column. The grand mean success rate for responses to visual targets was $19 \%$.

The average accuracy and the variability of individual responses to kinesthetic targets are illustrated in the right plot of Figure $4 B$. At $700 \mathrm{msec}$ after the onset of elbow rotation (on average, $530 \mathrm{msec}$ after response onset), the slope of the regression line was close to unity (slope $=1.162$ ). The grand mean success rate of responses to kinesthetic targets was $22 \%$ (bottom 

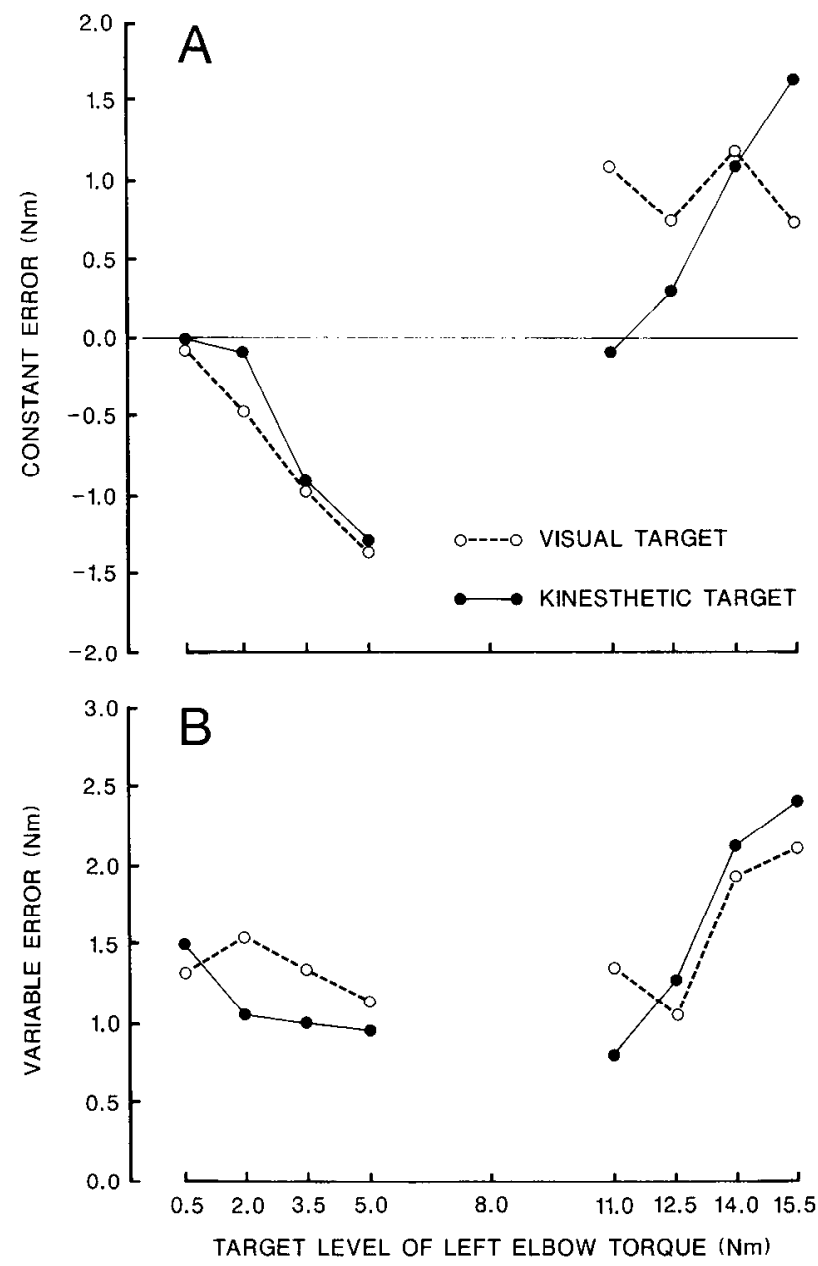

Figure 5. Accuracy of torque responses. The constant errors $(A)$ and variable errors $(B)$ at $700 \mathrm{msec}$ after the onset of target presentation were very similar for responses to visual targets (open symbols) and responses to kinesthetic targets (filled symbols).

of Table 1, right-most column). This success rate was not significantly different from the success rate for responses to visual targets $[t(10)=0.71, p>0.05]$.

The errors in responses to visual and kinesthetic targets were similar. The constant error (the difference between the target and average response levels of torque) and the variable error (the SD of the average response) were calculated for each subject and then averaged across all subjects (Fig. 5).

In most cases, the errors in responses to visual targets were not significantly different from the errors in responses to kinesthetic targets. A 3-way analysis of variance (type of target $x$ direction $\times$ target amplitude) showed that the type of target (visual or kinesthetic) had a significant effect on constant error only at one target amplitude, $F(1,5)=7.22, p<0.05$. For increases in torque from 8.0 to $11.0 \mathrm{Nm}$, the average response to visual targets overshot the target by about $1.0 \mathrm{Nm}$, whereas the average responses to kinesthetic targets had close to zero constant error. For variable error data (Fig. $5 B$ ), there were no significant differences between responses to visual and kinesthetic targets.

The effect of target amplitude on error was different for increases (Fig. 5, right) and decreases (Fig. 5, left) in torque. For both constant errors and variable errors, the analysis of variance showed a significant interaction between response direction and target amplitude. The constant errors of the decreases in torque were graded with target amplitude: There was more error for smaller changes in torque, $F(3,15)=16.03, p<0.001$. Also, the variable errors of the increases in torque were graded with target amplitude: There was more variability for larger increases in torque, $F(3,15)=10.44, p<0.001$.

\section{Discussion}

We have demonstrated that kinesthetic information about the direction and amplitude of target movement can be used as quickly and accurately as vision to parameterize new motor commands. Our results suggest that kinesthetic guidance of ongoing motor activity is not limited to reflexive compensation for perturbations.

\section{Comparison of kinesthetic and visual control}

Our results show that kinesthetic input can parameterize new motor commands faster than vision parameterizes new motor commands. Our results also show that kinesthesis and vision can work with similar accuracy. We base these conclusions on our comparisons of response latency, the latency of the control of response amplitude, and response accuracy.

For responses to kinesthetic targets and to visual targets, our estimates of response latency were consistent with past reports of kinesthetic and visual reaction times. In a previous experiment (Flanders et al., 1986), in which kinesthetic or visual input triggered a stereotypical increase in muscle activity to a single target amplitude, we found that kinesthetic response latency was about $110 \mathrm{msec}$ and visual response latency was about $190 \mathrm{msec}$. In our present study, in which kinesthetic or visual information produced either an increase or a decrease in muscle activity to 1 of 8 levels, kinesthetic response latency was about $150 \mathrm{msec}$ and visual response latency was about $250 \mathrm{msec}$. The relatively long response latencies in our present study are likely to have been caused by increased task complexity (Hyman, 1953; Henry and Rogers, 1960). The significant difference between visual and kinesthetic response latencies reported in both our earlier study (Flanders et al., 1986) and our present study is consistent with earlier literature (Woodworth and Schlosberg, 1954) and is presumably due to anatomical and physiological differences in processing delays in kinesthetic and visual pathways.

Responses both to kinesthetic targets and to visual targets became graded with target amplitude soon after the onset of the response. The time between response onset and the onset of significant amplitude control was not significantly different for responses to kinesthetic and visual targets. In contrast, the time between the onset of target presentation and the onset of significant amplitude control was significantly shorter for responses to kinesthetic targets. Presumably, this difference was due to inherent differences in processing delays (reaction time differences) rather than to differences in the mechanisms of response parameterization.

The accuracy of responses to kinesthetic targets was similar to the accuracy of responses to visual targets. The only significant difference in accuracy was for small increases in torque, where responses to kinesthetic targets had less constant error (Fig. 5A). This suggests that kinesthetic information may be more useful for fine adjustments in force.

A similarity between responses to visual and kinesthetic targets was that for both types of responses, subjects tended to 
overrespond when making small decreases in torque (Fig. 5A). This was because decreases in torque were produced by relaxation of both the biceps and triceps, and suggests that accurately producing very small decreases in muscle activity is difficult (see Fig. 2). If decreases in flexion torque had been produced by increases in extensor (triceps) activity instead of by decreases in both flexor and extensor activity, we would have expected the same results for both increases and decreases in torque.

Another similarity between responses to visual and kinesthetic targets is that for responses involving increases in torque, variable error was smaller for responses to smaller targets (Fig. 5 , right). This is consistent with past reports that small increases in torque are produced with less variability than are large increases in torque (Schmidt et al., 1979). We also demonstrated that, for responses involving decreases in torque, target amplitude did not have a significant effect on response variable error (Fig. 5, left). One possible explanation for this result is a cancellation of opposite effects: less variability for smaller levels of torque and less variability for smaller changes in torque.

The similarities in the latency of amplitude control and in response accuracy described above suggest that kinesthetic input can guide ongoing movement just as effectively as can visual input. This suggestion does not conflict with past reports that subjects prefer to use visual guidance (Klein, 1977), because visual input is necessary for sensing the distance of remote (unfelt) targets. Because of this advantage of visual sensation, the nervous system may choose visual guidance over kinesthetic guidance when given a choice. Kinesthetic guidance may, however, be useful in situations such as manipulating an object in the dark or holding a moving object when visual attention is directed elsewhere.

\section{Kinesthetic guidance}

We have distinguished between 2 forms of kinesthetic guidance: reflexive compensation for perturbations and parameterization of new motor commands based on new target information. In past studies, most investigators have perturbed movements that were directed at a fixed goal and have stressed the short latency and reflexive nature of compensatory motor activity (e.g., Cole and Abbs, 1987). Some investigators have also suggested that short-latency, reflexive changes in motor activity may be followed, at a longer latency, by a reparameterization of new motor commands (Day and Marsden, 1982; Johansson and Westling, 1987). It is possible that the neural basis of this longer-latency mechanism for compensation to perturbations is identical to the neural basis of the mechanism for acquiring new targets that we have described in our present study.

Our subjects used kinesthetic input from the right arm to produce accurate increases or decreases in voluntary motor commands to the muscles of the left elbow (as shown by the increases and decreases in EMG in Fig. 2). We assume that these responses were produced by the parameterization of voluntary motor commands rather than by nonautogenic reflexes because, although our subjects were instructed to respond as quickly as possible, they responded after typical voluntary reaction times (Flanders, et al., 1986; see also Cole and Abbs, 1987; Johansson and Westling, 1987). Although we used a bimanual task to describe a kinesthetic control mechanism, this mechanism may prove to be of general utility in other voluntary motor activities. Target information sensed by kinesthetic receptors in the right elbow might be equally useful in guiding movement of the eyes, the head, or even the right elbow itself.

\section{References}

Abbs, J. H., and V. L. Gracco (1984) Control of complex motor gestures: Orofacial muscle responses to load perturbations of lip during speech. J. Neurophysiol. 51: 705-723.

Chernikoff, R., and F. V. Taylor (1952) Reaction time to kinesthetic stimulation resulting from sudden arm displacement. J. Exp. Psychol. 43: $1-8$.

Cole, K. J., and J. H. Abbs (1987) Kinematic and electromyographic responses to perturbation of a rapid grasp. J. Neurophysiol. 57: 14981510 .

Cordo, P. J. (1987) Mechanisms controlling accurate changes in elbow torque in humans. J. Neurosci. 7: 432-442.

Crago, P. E., J. C. Houk, and Z. Hasan (1976) Regulatory actions of human stretch reflex. J. Neurophysiol. 39: 925-935.

Day, B. L., and C. D. Marsden (1982) Accurate repositioning of the human thumb against unpredictable dynamic loads is dependent upon peripheral feedback. J. Physiol. (Lond.) 327: 393-407.

De Domenico, G., and D. I. McCloskey (1987) Accuracy of voluntary movements at the thumb and elbow joints. Exp. Brain Res. 65: $471-$ 478 .

Dufresne, J. R., V. S. Gurfinkel, J. F. Soechting, and C. A. Terzuolo (1978) Response to transient disturbances during intentional forcarm flexion in man. Brain Res. 150: 103-115.

Evarts, E. V., and W. J. Vaughn (1978) Intended arm movements in response to externally produced arm displacements in man. In $\mathrm{Ce}$ rebral Motor Control in Man: Long Loop Mechanisms (Prog. Clin. Neurophysiol., Vol. 4), J. E. Desmedt, ed., pp. 178-192, Karger, Basel.

Flanders, M., and P. J. Cordo (1987) Quantification of peripherally induced reciprocal activation during voluntary muscle contraction. Electroencephalogr. Clin. Neurophysiol. 67: 389-394.

Flanders, M., P. J. Cordo, and J. G. Anson (1986) Interaction between visually and kinesthetically triggered voluntary responses. J. Motor Behav. 18: 427-448.

Gordon, J., and C. Ghez (1987) Trajectory control in targeted force impulses: III. Compensatory adjustments for initial errors. Exp. Brain Rcs. 67: 253-269.

Hammond, P. H. (1954) Involuntary activity in biceps following the sudden application of velocity to the abducted forearm. J. Physiol. (Lond.) 127: 23P-25P.

Henry, F. M., and D. E. Rogers (1960) Increased response latency for complicated movements and a "memory drum" theory of neuromotor reaction. Res. Q. 31: 448-458.

Hyman, R. (1953) Stimulus information as a determinant of reaction time. J. Exp. Psychol. 45: 188-196.

Johansson, R. S., and G. Westling (1984) Roles of glabrous skin receptors and sensorimotor memory in automatic control of precision grip when lifting rougher or more slippery objects. Exp. Brain Res. 56: $550-564$.

Johansson, R. S., and G. Westling (1987) Signals in tactile afferents from the fingers eliciting adaptive motor responses during precision grip. Exp. Brain Res. 66: 141-154.

Klein, R. M. (1977) Attention and visual dominance: A chronometric analysis. J. Exp. Psychol. [Hum. Percept.] 3: 365-378.

Marsden, C. D., P. A. Merton, and H. B. Morton (1976) Servo action in the human thumb. J. Physiol. (Lond.) 257: 1-44.

Pélisson, D., C. Prablanc, M. A. Goodale, and M. Jeannerod (1986) Visual control of reaching movements without vision of the limb: II. Evidence of fast unconscious processes correcting the trajectory of the hand to the final position of a double-step stimulus. Exp. Brain Res. 62: 303-311.

Schmidt, R. A., H. Zelaznik, B. Hawkins, J. S. Frank, and J. T. Quinn (1979) Motor-output variability: A theory for the accuracy of rapid motor acts. Psychol. Rev. 86: 415-451.

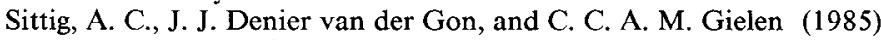
Separate control of arm position and velocity demonstrated by vibration of muscle tendon in man. Exp. Brain Res. 60: 445-453.

Sittig, A. C., J. J. Denier van der Gon, and C. C. A. M. Gielen (1987) The contribution of afferent information on position and velocity to the control of slow and fast human forearm movements. Exp. Brain Res. 67: 33-40.

Snedecor, G. W., and W. G. Cochran (1971) Statistical Methods, 6th ed., Iowa State University Press, Ames, IA.

Woodworth, R. S., and H. Schlosberg (1954) Experimental Psychology, Holt, New York. 